\title{
La fratrie comme ressource \\ Le rôle des aînés dans les parcours scolaires des enfants de familles nombreuses
}

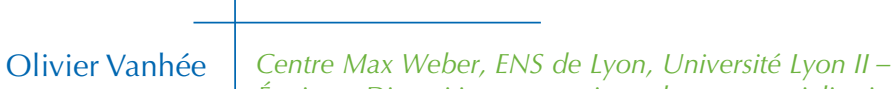 \\ Équipe "Dispositions, pouvoirs, cultures et socialisations \\ Géraldine Bois Centre Max Weber, ENS de Lyon, Université Lyon II - \\ Gaële Henri-Panabière $\quad \begin{aligned} & \text { Équipe «Dispositions, pouvoirs, cultures et socialisa } \\ & \text { Université Paris Descartes, Sorbonne Paris Cité, }\end{aligned}$ \\ Centre de recherche sur les liens sociaux. \\ Martine Court Université Blaise Pascal (Clermont-Ferrand II) - LAPSCO/CNRS. \\ Julien Bertrand Université Paul Sabatier (Toulouse III) - Équipe SOI \\ (PRISSMH) et Centre Max Weber, ENS de Lyon, Équipe \\ "Dispositions, pouvoirs, cultures et socialisations».
}

Mots-clés : Fratrie - Familles nombreuses - Parcours scolaire.

De nombreuses enquêtes statistiques montrent que le fait de grandir au sein d'une famille nombreuse a un effet négatif sur les devenirs scolaires. Une enquête par entretiens auprès d'une vingtaine de ces familles permet de mettre en évidence comment la fratrie peut, au contraire, devenir une ressource, en particulier dans les familles les plus démunies en capitaux culturels. L'article propose ainsi d'analyser les modalités et les effets de l'accompagnement scolaire et culturel assuré par des aînés de familles nombreuses. S'il est possible d'observer, sous certaines conditions, des effets scolaires bénéfiques pour les cadets, on peut noter également des effets " en retour " de cette intervention fraternelle sur la scolarité des aînés eux-mêmes et sur les pratiques éducatives des parents.

es enquêtes statistiques réalisées ces quinze dernières années montrent que le fait de grandir dans une famille nombreuse a un effet négatif sur les parcours scolaires des enfants (Barnet-Verzat et Wolff, 2003 ; Caillé et O'Prey, 2002 ; Caillé et Rosenwald, 2006). Si ce type de configuration familiale est marqué par une nette surreprésentation des familles populaires $[47 \%$ des pères de familles de quatre enfants et plus sont ouvriers, $11 \%$ sont employés (1)], ces travaux soulignent l'existence d'un effet négatif de la taille de la fratrie à origines sociales équivalentes. Ainsi, toutes choses égales par ailleurs, les enfants issus d'une famille de trois enfants et plus sont plus nombreux que les autres à redoubler à l'école primaire et au collège. L'âge auquel ils terminent leurs études est également plus bas en moyenne que celui des enfants nés dans une famille de taille plus réduite.
Cet effet négatif de la taille de la fratrie sur la scolarité peut résulter de différents processus, non exclusifs les uns des autres. Il peut trouver son principe dans une altération des conditions de vie matérielles des familles liée à l'augmentation du nombre d'enfants (Bertrand et al., 2012 a) ; il peut également résulter d'une diminution du suivi scolaire, de l'accompagnement culturel ou encore du contrôle des activités enfantines assurés par les parents, ceux-ci devenant moins disponibles au fur et à mesure de l'agrandissement de la fratrie (Bertrand et al., 2012 b).

Pour autant, l'accroissement de la taille de la famille n'altère pas nécessairement les conditions de scolarité des enfants. Certaines familles nombreuses disposent, en effet, de ressources susceptibles de limiter les effets négatifs de cet accroissement, en leur permettant en particulier de compenser, à des degrés divers, la diminution de la disponibilité parentale. Parmi ces ressources, les ressources économiques sont les plus évidentes. En autorisant les parents à "s'acheter du temps»-par exemple, par l'embauche d'une femme de ménage (Chauvel, 2001) ou à recourir à des relais éducatifs payants (cours particuliers, internat, etc.), elles permettent de pallier, au moins partiellement, cette diminution. Les ressources économiques ne sont cependant pas les seules susceptibles de jouer un tel rôle. Dans certaines familles, I'affaiblissement de la disponibilité parentale peut également être compensé par une prise en charge des cadets par les aînés. Alors que les familles de classes moyennes et supérieures sont, par définition, mieux dotées économiquement, cette ressource particulière que

(1) $17 \%$ des pères de ces familles occupent des emplois de cadres et professions intellectuelles supérieures, $13 \%$ de professions intermédiaires, $8 \%$ d'artisans et $2 \%$ d'agriculteurs (Blanpain, 2007). 
constitue l'intervention des aînés semble quant à elle plus spécifique aux classes populaires. Dans leur scolarité, les enfants d'ouvriers reçoivent, en effet, plus souvent que les autres le soutien d'un membre de la famille qui n'est pas I'un de leurs parents (Poullaouec, 2010).

L'objectif de cet article est d'analyser comment cette prise en charge des cadets par les aînés au sein des familles nombreuses peut constituer une ressource d'un point de vue scolaire, pour ces cadets, mais aussi pour les aînés eux-mêmes. On s'intéresse ici à deux modalités de cette prise en charge : le suivi de la scolarité et l'accompagnement « lectoral ». Tel qu'on l'entend ici, le suivi de la scolarité regroupe plusieurs pratiques: I'aide aux devoirs, le suivi des résultats et de l'orientation, et l'investissement dans les établissements scolaires (présence aux réunions avec les enseignants, participation aux sorties, etc.). En ce qui concerne l'accompagnement " lectoral », on s'intéresse à la fois aux pratiques d'accompagnement en tant que telles (accompagnement à la bibliothèque et conversations autour de la lecture) et aux pratiques d'initiation (lecture d'histoires pendant l'enfance, cadeaux de livres ou d'autres imprimés, incitations à lire, etc.).

Le choix de ces deux ensembles de pratiques résulte de leur association étroite avec la réussite scolaire. Si toutes les formes de suivi ne sont pas corrélées aux résultats scolaires ( $c^{\prime}$ est le cas notamment de l'aide aux devoirs), plusieurs enquêtes montrent que l'implication des parents dans la scolarité des enfants, sous forme de suivi des résultats et de conversations régulières, est positivement liée à la réussite de ces derniers et à leur intérêt pour l'école (intérêt qui se manifeste par un investissement dans le travail scolaire à la maison, de bonnes relations avec les enseignants, des projets d'études longues, etc.) (Lahire, 1992 ; Terrail, 1992). De même, de nombreuses enquêtes ont mis en évidence les effets positifs de la lecture d'un point de vue scolaire (Laurens, 1992 ; Lahire, 1995 ; Michaudon, 2001). Même si les habitudes de lecture constituées au sein de la famille ne correspondent pas toujours aux attendus de l'école (Renard, 2008) et si le rendement scolaire des lectures s'affaiblit avec l'avancée dans le cycle des études (Coulangeon, 2007), l'intensité et la précocité de cette pratique sont bel et bien liées à une meilleure réussite. Certaines pratiques d'accompagnement scolaire et « lectoral » participent ainsi à un mode de socialisation familial scolairement rentable.

Cet article décrit en premier lieu les modalités et les conditions de la participation des aînés à la socialisation de leurs frères et sœurs dans les familles nombreuses étudiées. Puis, il interroge les effets bénéfiques de cette participation en analysant, $d$ 'une part, les effets sur la scolarité des cadets de ces familles et, d'autre part, les effets «en retour», $c^{\prime}$ est-à-dire les répercussions de cette intervention fraternelle sur la scolarité des aînés et sur les pratiques éducatives des parents.

\section{Modalités et conditions d'intervention des aînés}

L'action des aînés peut prendre plusieurs formes et s'articuler de différentes manières avec celle des parents. Dans une partie des familles, les parents, scolairement dotés, ont mis en place un accompagnement scolaire et " lectoral » pour leurs aînés. Lorsque la disponibilité des parents est affaiblie par l'agrandissement de la fratrie, les aînés peuvent alors prendre leur relais. Pour une autre partie des familles interrogées, les pères et les mères sont moins diplômés et n'ont pas toujours pu accompagner les aînés dans leur travail scolaire ou dans leurs lectures. Ce sont alors souvent ces aînés qui portent les capitaux scolaires les plus importants et jouent, au sein de la famille, un rôle d'interprètes de la culture scolaire pour leurs cadets.

\section{Des aînés relais éducatifs des parents}

Dans certaines familles, l'intervention d'un ou de plusieurs aîné(s) vient suppléer à la moindre disponibilité parentale, et permet ainsi d'assurer une continuité de l'encadrement familial lorsque celui-ci est moins intense pour les cadets. Cette intervention des aînés comme relais éducatifs s'observe dans différents milieux sociaux, mais toujours dans des familles où la mère au moins détient des capitaux scolaires qui lui ont permis de mettre en œuvre, par le passé, un mode de socialisation scolairement rentable. Dans la famille Fernel (famille biparentale, cinq enfants, père chauffeur routier, mère inactive, bachelière) par exemple, la mère a consacré beaucoup de temps à l'encadrement scolaire et « lectoral » de sa fille aînée Émilie, mais avec ses cadets, cet encadrement est devenu moins soutenu. Alors qu'elle était présente presque quotidiennement pour aider son aînée dans ses devoirs, et ce jusqu'en classe de cinquième au collège (moment où Émilie s'est mise à travailler seule), elle a suivi de manière beaucoup plus lâche les devoirs de ses cadets. De la même façon, si Émilie et sa cadette immédiate ont bénéficié de lectures quotidiennes $d^{\prime}$ histoires de la part de leur mère pendant de nombreuses années (jusqu'à l'âge de 8 ans pour Émilie, 4 ans pour sa sœur), ce n'est pas le cas de leurs trois cadets. L'arrêt de cette pratique correspond précisément à la naissance des benjamines (deux jumelles nées deux ans après le troisième enfant), moment où $M^{\text {me }}$ Fernel était « trop fatiguée » pour lire, pour elle-même comme pour ses enfants ("Plus il y avait de bébés, plus c'était difficile d'avoir des moments », explique-t-elle). 
Au-delà de cet aspect de l'histoire familiale (une naissance gémellaire), si $M^{\text {me }}$ Fernel a été nettement moins disponible pour ses cadets que pour ses aînées, c'est aussi pour deux autres raisons : d'une part, parce que les naissances des enfants ont été rapprochées (cinq enfants en sept ans) et, d'autre part, parce que les parents n'ont jamais eu recours à des aides extérieures payantes pour les seconder dans les tâches domestiques et éducatives.

Dans la famille Fernel, cette diminution de I'encadrement maternel a cependant été compensée, en partie, par des interventions d'Émilie auprès des autres enfants de la fratrie. Lorsque celle-ci était en classe de CM1 à l'école primaire, elle s'est en effet mise à lire des histoires le soir à ses cadets, prenant ainsi le relais de sa mère qui le faisait de moins en moins souvent (à ce moment-là, la fratrie comporte cinq enfants, âgés de 9 ans à 2 ans). De même, lorsqu'elle était en classe de cinquième, Émilie a pris le relais de sa mère pour l'encadrement des devoirs des cadets (ceux-ci sont alors à l'école primaire en classes de CE2 et de CP et à l'école maternelle). "La première, explique $M^{\text {me }}$ Fernel, je travaillais beaucoup avec elle étant donné qu'elle était seule. Et puis, maintenant, ce qui est bien, c'est qu'ils s'entraident les uns les autres. (...) Je m'intéresse toujours à ce qu'ils font, je mets toujours mon nez au milieu, mais... ils se font réciter les leçons, quel que soit le niveau ».

Dans les familles des catégories supérieures, mieux dotées que la famille Fernel d'un point de vue économique et culturel, cette intervention fraternelle semble à la fois plus limitée (elle concerne principalement les enfants qui éprouvent le plus de difficultés) et moins nécessaire. Cette nécessité est moindre pour deux raisons. D'abord, les moyens économiques de ces familles permettent aux parents de se libérer du temps pour le travail éducatif, à la fois par le recours à des aides rémunérées (soutien scolaire, internat, aides ménagères) et par la possibilité (qui concerne avant tout les mères, mais aussi parfois les pères) d'interrompre ou de réduire leur activité professionnelle. Ainsi, dans la famille Wemel (famille biparentale et recomposée, quatre enfants, père inspecteur des impôts, mère agent des impôts, bachelière), les deux garçons ont tous deux été moins autonomes dans leur travail scolaire que les deux filles ("C'était pas forcément très naturel chez eux de faire leurs devoirs tout seuls ») mais, parmi ces garçons, c'est le benjamin (Geoffroy) qui a été le plus suivi scolairement par ses parents. Cette situation a été rendue possible par le fait que $M^{\text {me }}$ Wemel a travaillé à temps partiel pendant plusieurs années et, surtout, par le fait qu'elle a pu prendre sa retraite à l'âge de 47 ans, lorsque Geoffroy entrait au collège en classe de quatrième (à cette date, l'autre garçon de la fratrie avait, en revanche, terminé ses études). Ainsi, alors que d'après leur fille Justine, "[s]es parents ont un peu lâché I'affaire avec [s]on grand frère au collège-lycée", ils ont davantage suivi la scolarité du benjamin. Au moment de l'enquête, celui-ci est au lycée en terminale $S$, et on peut supposer que, sans l'encadrement serré de ses parents, il n'aurait peut-être pas réussi à intégrer cette filière scientifique.

La deuxième raison pour laquelle l'intervention fraternelle est moins nécessaire dans les familles de classes supérieures réside dans le fait que, dans ces familles, les enfants ont souvent intériorisé de manière précoce la nécessité du travail scolaire. Dans la plupart des cas, ils travaillent d'eux-mêmes et seuls depuis leur scolarité élémentaire. Le mode $d^{\prime}$ accompagnement scolaire des parents se fonde sur un idéal $d^{\prime}$ « autonomie » et se traduit généralement par un suivi assez "lâche » des devoirs, l'aide au travail scolaire n'étant fournie aux enfants qu'à leur demande. Dans la famille Denissov (famille biparentale, cinq enfants, père instituteur, mère infirmière), les parents déclarent ainsi avoir peu contrôlé les devoirs de leurs enfants et s'être appuyés essentiellement sur leur autocontrainte et leur aisance scolaire. Comme l'indique $\mathrm{M}^{\mathrm{me}}$ Denissov, " c'était des enfants qui étaient suffisamment consciencieux pour faire ce qu'on leur demandait de faire. (...) Et suffisamment intelligents pour suivre comme ça ». Cela étant, $M$. et $\mathrm{M}^{\mathrm{me}}$ Denissov ont également eu recours à des aides extérieures payantes à certains moments critiques de la scolarité de leurs enfants. Ils ont inscrit leur deuxième fils dans un internat privé lorsque celui-ci a rencontré des difficultés, et ils ont financé des cours particuliers pour chacun de leurs enfants dans des matières où ils éprouvaient des difficultés.

Si les interventions des aînés auprès de leurs cadets sont plus limitées dans ces familles de classes supérieures, elles ne sont cependant pas inexistantes. Dans ces familles, les interventions fraternelles viennent essentiellement redoubler celles des parents. C'est ce que l'on observe, par exemple, dans la famille Dallier (famille biparentale, cinq enfants, père chef $d^{\prime}$ entreprise, mère sage-femme) où le deuxième enfant de la fratrie (Léo) est le seul à connaître des difficultés scolaires (au moment de I'enquête, il redouble la classe de troisième). Erwan (I'aîné) relaie auprès de Léo les mises en garde qui lui sont aussi adressées par leurs parents; il vient donc renforcer le soutien que ces derniers lui apportent. Selon Erwan, le fait de ne pas avoir le statut de parent lui permet d'être plus facilement entendu par son frère cadet : "Avec Léo on discute beaucoup (de sa scolarité) parce que, là, il arrive à un moment où il doit s'orienter, donc c'est super important pour ce qu'il va faire après. Donc j'en discute avec lui, parce que mes parents lui en parlent, que c'est important etc., mais (c'est bien) qu'il ait un avis aussi... enfin, plus proche que les parents». 


\section{L'enquête}

Les analyses présentées dans cet article s'appuient sur les résultats d'une enquête financée par la Caisse nationale des Allocations familiales dans le cadre de l'appel à projets " Destin social des fratries » (1). Cette enquête visait à mieux comprendre le lien entre les parcours scolaires et la taille de la fratrie, en mettant en évidence de quelles manières et sous quelles conditions la taille de la fratrie agit sur les formes de socialisation familiale et, partant, sur les parcours scolaires des enfants de ces familles. Une enquête par entretiens a ainsi été réalisée en 2010 et 2011 auprès de vingt-deux familles d'au moins quatre enfants (voir le tableau ci-contre). Dans la plupart de ces familles, deux entretiens ont été réalisés, le premier avec l'un des enfants, âgé de 18 ans à 27 ans et ayant terminé ses études secondaires, le second avec l'un des parents ou, à défaut, I'un de ses aînés (2). N'ont été retenus dans la population d'enquête que des jeunes ayant vécu avec au moins trois germains pendant une durée relativement longue (cinq années au moins). En termes de socialisation, ce qui importe en effet n'est pas tant d'être issu d'une famille nombreuse que d'avoir grandi pendant plusieurs années au sein d'une famille nombreuse. La sélection de la population s'est appliquée à faire varier les positions sociales des familles et, sans prétendre à la représentativité, à conserver la surreprésentation des membres des classes populaires observée dans ce type de familles. Douze pères appartiennent ainsi aux classes populaires, cinq aux classes moyennes et cinq aux classes supérieures. De manière secondaire, cette sélection a également veillé à inclure des jeunes des deux sexes et ayant des rangs de naissance différents au sein de leur fratrie.

Les entretiens visaient à explorer des dimensions de la socialisation familiale particulièrement significatives du point de vue des performances scolaires : les conditions matérielles d'existence, l'accompagnement scolaire et culturel des enfants, la régulation de la vie domestique et, enfin, les projets familiaux et professionnels des jeunes adultes interrogés.

(1) Bertrand J., Bois G., Court M., Henri-Panabière G. et Vanhée O., 2012, "Les parcours scolaires d'enfants de familles nombreuses: I'analyse des processus de socialisation », rapport de recherche pour la Caisse nationale des Allocations familiales, Centre Max Weber, université Lyon 2.

(2) Dans quelques familles, un seul entretien a pu être réalisé, soit avec l'enfant, soit avec son parent.

\section{Des aînés interprètes de la culture scolaire}

Lorsque les parents sont peu dotés scolairement, ils ne possèdent généralement pas les ressources leur permettant de suivre le travail scolaire de leurs enfants, de les guider dans leur orientation et de les initier à la culture écrite. Dans certains cas, les aînés peuvent cependant se substituer aux parents dans ces différents rôles. Lorsqu'ils ont eux-mêmes été socialisés longuement au sein de l'institution scolaire, ils sont en effet en mesure d'assurer un certain accompagnement scolaire et culturel auprès de leurs cadets.
Dans certaines familles, ce sont ainsi les aînés qui encadrent le travail scolaire des autres enfants de la fratrie. Dans la famille Kharmaz, notamment, (famille biparentale, cinq enfants, père chauffeur poids-lourds, mère inactive puis femme de ménage), l'aînée des filles, Fatima, s'est beaucoup impliquée dans cet encadrement (elle est la deuxième enfant de sa fratrie). Elle-même a connu un parcours scolaire moyen (elle a obtenu un baccalauréat technologique à l'âge de 20 ans), mais elle dit avoir aimé l'école et fait preuve de beaucoup de bonne volonté scolaire. Elle aurait souhaité suivre une filière $S$ puis des études de médecine, mais ses parents ne lui ont pas donné la possibilité de le faire. Dès l'âge de 13 ans, Fatima a pris en charge le suivi du travail scolaire de ses cadets. Elle a appris à lire à sa sœur Leïla (avec qui elle a huit ans d'écart) avant son entrée au cours préparatoire, "pour lui donner la chance (...) d'être un peu plus en avance par rapport aux autres ». Elle a ensuite encadré ses devoirs quotidiennement, en primaire et au collège ( SColairement, c'est moi qui l'ai prise en main »). Depuis qu'elle a quitté le domicile parental, elle continue à suivre à distance la scolarité de sa jeune sœur, ainsi que celle de son frère Yacine. Pendant I'année de l'enquête (Yacine est alors en classe de troisième), elle est ainsi allée rencontrer l'un de ses professeurs "pour savoir comment ça se passait ". Yacine la tient, en outre, informée de ses résultats. À la fin de l'entretien avec elle, il l'appelle justement pour lui annoncer qu'il a eu 19 à son rapport de stage. Elle le félicite alors chaleureusement, puis précise à l'enquêtrice qu'elle « l'encourage » et qu'elle " essaye de lui donner beaucoup d'importance par rapport à ça ».

Dans certains cas, les aînés constituent également une ressource pour leurs cadets du point de vue de l'orientation. Premiers de leur famille à avoir fréquenté le lycée, ils connaissent généralement mieux que leurs parents le système éducatif secondaire et sont en mesure de se substituer à eux pour aider leurs cadets dans leurs choix d'orientation. Dans la famille Bertou (famille biparentale, quatre enfants, père ouvrier qualifié, mère assistante maternelle), les parents n'ont pas fréquenté le lycée d'enseignement général [le père détient un brevet d'enseignement professionnel (BEP), la mère le brevet d'études du premier cycle du second degré (BEPC)] et ils connaissent peu son fonctionnement. Au moment où Nicolas (troisième enfant de la fratrie) a dû faire ses choix d'orientation à ce niveau, c'est donc avant tout vers son frère aîné qu'il s'est tourné. Celui-ci l'a conseillé pour choisir ses options au lycée en classe de seconde, puis sa série de baccalauréat, et enfin sa filière de brevet de technicien supérieur (BTS). De même, ce sont les moqueries de sa sœur aînée sur les "Troisièmes techno » qui ont amené Nicolas à prendre conscience de la mauvaise réputation de cette filière scolaire quand il 


\begin{tabular}{|c|c|c|c|c|c|c|}
\hline \multicolumn{7}{|c|}{ Les caractéristiques sociales } \\
\hline \multicolumn{7}{|c|}{ des enquêtés } \\
\hline Enfant enquêté & Âge & Situation actuelle & \begin{tabular}{|c|} 
Rang \\
dans la \\
fratrie $(*)$
\end{tabular} & $\begin{array}{l}\text { Profession } \\
\text { paternelle } \\
\text { (diplôme) }\end{array}$ & $\begin{array}{l}\text { Profession } \\
\text { maternelle } \\
\text { (diplôme) }\end{array}$ & $\begin{array}{l}\text { Structure } \\
\text { familiale }\end{array}$ \\
\hline Sofiane Badji & 21 ans & $\begin{array}{l}\text { Baccalauréat } \\
\text { professionnel } \\
\text { (en cours) }\end{array}$ & $1 / 6$ & $\begin{array}{l}\text { Employé de } \\
\text { restauration } \\
\text { (baccalauréat) }\end{array}$ & $\begin{array}{l}\text { Inactive (scolarisée } \\
\text { jusqu'au collège, en } \\
\text { classe de troisième) }\end{array}$ & $\begin{array}{l}\text { Biparentale } \\
\text { recomposée puis } \\
\text { monoparentale }\end{array}$ \\
\hline Nora Bedjaoui & 23 ans & $\begin{array}{l}\text { En recherche } \\
\text { d'emploi (master) }\end{array}$ & $2 / 7$ & $\begin{array}{l}\text { Ouvrier } \\
\text { (certificat d'études) }\end{array}$ & $\begin{array}{l}\text { Employée puis } \\
\text { femme de ménage } \\
\text { (CAP) }\end{array}$ & Biparentale \\
\hline Marion Bernier & 20 ans & $\begin{array}{l}\text { Licence } 2 \\
\text { " Médecine » } \\
\text { (en cours) }\end{array}$ & $3 / 7$ & $\begin{array}{l}\text { Directeur } \\
\text { d'entreprise } \\
\text { (école d'ingénieur) }\end{array}$ & $\begin{array}{l}\text { Kinésithérapeute } \\
\text { (DE) }\end{array}$ & Biparentale \\
\hline Nicolas Bertou & 23 ans & $\begin{array}{l}\text { Technicien supérieur } \\
\text { (BTS) }\end{array}$ & $3 / 4$ & $\begin{array}{l}\text { Ouvrier qualifié } \\
\text { (BEP) }\end{array}$ & $\begin{array}{l}\text { Assistante maternelle } \\
\text { (BEPC) }\end{array}$ & Biparentale \\
\hline Héléna Chautard & 18 ans & $\begin{array}{l}\text { Licence } 2 \\
\text { "Biologie » } \\
\text { (en cours) }\end{array}$ & $4 / 4$ & $\begin{array}{l}\text { Jardinier (ancien } \\
\text { parachutiste, peu } \\
\text { scolarisé) }\end{array}$ & Aide-soignante & $\begin{array}{l}\text { Biparentale } \\
\text { recomposée puis } \\
\text { monoparentale }\end{array}$ \\
\hline Bérangère Cotti & 22 ans & $\begin{array}{l}\text { École des Beaux-Arts } \\
\text { (en cours) }\end{array}$ & $6 / 6$ & $\begin{array}{l}\text { Ouvrier en invalidité } \\
\text { (peu scolarisé) }\end{array}$ & $\begin{array}{l}\text { Caissière } \\
\text { (peu scolarisée) }\end{array}$ & $\begin{array}{l}\text { Biparentale } \\
\text { recomposée puis } \\
\text { monoparentale }\end{array}$ \\
\hline Erwan Dallier & 20 ans & $\begin{array}{l}\text { École d'ingénieur } \\
\text { (en cours) }\end{array}$ & $1 / 5$ & $\begin{array}{l}\text { Directeur } \\
\text { d'entreprise (BTS) }\end{array}$ & Sage-femme (DE) & Biparentale \\
\hline Emma Denissov & 22 ans & Master 1 (en cours) & $3 / 5$ & $\begin{array}{l}\text { Instituteur } \\
\text { (école normale) }\end{array}$ & Infirmière (DE) & Biparentale \\
\hline Marc Djelloul & 24 ans & $\begin{array}{l}\text { En formation en } \\
\text { mission locale (CAP) }\end{array}$ & $1 / 5$ & Employé & $\begin{array}{l}\text { Femme de ménage } \\
\text { (peu scolarisée) }\end{array}$ & $\begin{array}{l}\text { Biparentale et } \\
\text { recomposée }\end{array}$ \\
\hline Joel Dos Santos & 20 ans & $\begin{array}{l}\text { Licence } 1 \\
\text { "Anthropologie » } \\
\text { (en cours) }\end{array}$ & $5 / 5$ & $\begin{array}{l}\text { Artisan } \\
\text { (peu scolarisé) }\end{array}$ & $\begin{array}{l}\text { Inactive (employée, } \\
\text { ouvrière) }\end{array}$ & $\begin{array}{l}\text { Biparentale et } \\
\text { recomposée }\end{array}$ \\
\hline Marie-Aline Dumont & 21 ans & $\begin{array}{l}\text { École d'infirmière } \\
\text { (en cours) }\end{array}$ & $3 / 7$ & $\begin{array}{l}\text { Moniteur } \\
\text { d'auto-école (BEP) }\end{array}$ & $\begin{array}{l}\text { Inactive } \\
\text { (employée, bac B) }\end{array}$ & Biparentale \\
\hline Émilie Fernel & 18 ans & BEP & $1 / 5$ & $\begin{array}{l}\text { Chauffeur-livreur } \\
\text { (BEP) }\end{array}$ & $\begin{array}{l}\text { Inactive (ménages, } \\
\text { secrétariat, bac G) }\end{array}$ & Biparentale \\
\hline Camille Foucart & 20 ans & $\begin{array}{l}\text { Licence } 3 \\
\text { " Économie » } \\
\text { (en cours) }\end{array}$ & $2 / 5$ & $\begin{array}{l}\text { Technicien géomètre } \\
(\text { bac }+2)\end{array}$ & $\begin{array}{l}\text { Laïque en mission } \\
\text { d'église, secrétaire } \\
(\text { bac }+2)\end{array}$ & Biparentale \\
\hline Samira Harouz & 22 ans & $\begin{array}{l}\text { Formation secrétariat } \\
\text { médical (en cours) }\end{array}$ & $4 / 8$ & $\begin{array}{l}\text { Ouvrier retraité } \\
\text { (non scolarisé) }\end{array}$ & $\begin{array}{l}\text { Femme de ménage } \\
\text { (peu scolarisée) }\end{array}$ & $\begin{array}{l}\text { Biparentale } \\
\text { recomposée puis } \\
\text { monoparentale }\end{array}$ \\
\hline Morgane Joubert & 20 ans & $\begin{array}{l}\text { Licence } 1 \\
\text { «Sociologie » } \\
\text { (en cours) }\end{array}$ & $1 / 4$ & $\begin{array}{l}\text { Echafaudeur } \\
\text { (aucun diplôme) }\end{array}$ & Secrétaire (bac F8) & Biparentale \\
\hline Leïla Kharmaz & 18 ans & $\begin{array}{l}\text { Licence } 1 \\
\text { "Psychologie " } \\
\text { (en cours) }\end{array}$ & $4 / 5$ & $\begin{array}{l}\text { Chauffeur poids } \\
\text { lourds, en invalidité } \\
\text { (a arrêté l'école à } \\
\text { l'âge de } 14 \text { ans) }\end{array}$ & $\begin{array}{l}\text { Sans profession } \\
\text { (ménages, peu scola- } \\
\text { risée) }\end{array}$ & Biparentale \\
\hline Laurent Latouche & 24 ans & $\begin{array}{l}\text { Ingénieur (école } \\
\text { d'ingénieur) }\end{array}$ & $4 / 4$ & $\begin{array}{l}\text { Chargé d'études } \\
(\text { bac }+4)\end{array}$ & Professeure agrégée & $\begin{array}{l}\text { Biparentale puis } \\
\text { monoparentale }\end{array}$ \\
\hline Geneviève Lemaire & 27 ans & $\begin{array}{l}\text { Professeure agrégée } \\
(\text { bac }+5)\end{array}$ & $1 / 5$ & Médecin hospitalier & Médecin hospitalier & Biparentale \\
\hline Nadia Mejri & 24 ans & Employée (bac + 3) & $6 / 6$ & $\begin{array}{l}\text { Ouvrier retraité } \\
\text { (peu scolarisé) }\end{array}$ & $\begin{array}{l}\text { Agent hospitalier } \\
\text { (scolarisée jusqu'en } \\
\text { classe de quatrième) }\end{array}$ & $\begin{array}{l}\text { Biparentale } \\
\text { et recomposée }\end{array}$ \\
\hline Alexandre Pauvert & 24 ans & $\begin{array}{l}\text { Salarié agricole } \\
\text { (BTS) }\end{array}$ & $1 / 4$ & $\begin{array}{l}\text { Chef d'exploitation } \\
\text { (BTS) }\end{array}$ & $\begin{array}{l}\text { Conjointe collabora- } \\
\text { trice, formatrice (BTS) }\end{array}$ & Biparentale \\
\hline François Pradier & 20 ans & BTS (en cours) & $1 / 6$ & Artisan (BTS) & $\begin{array}{l}\text { Formatrice en } \\
\text { éducation civique } \\
\text { et religieuse (bac) }\end{array}$ & Biparentale \\
\hline Justine Wemel & 20 ans & $\begin{array}{l}\text { Licence } 1 \text { " Droit » } \\
\text { (en cours) }\end{array}$ & $3 / 4$ & $\begin{array}{l}\text { Inspecteur des } \\
\text { impôts (bac }+3 \text { ) }\end{array}$ & $\begin{array}{l}\text { Agent des impôts } \\
\text { (bac) }\end{array}$ & $\begin{array}{l}\text { Biparentale } \\
\text { et recomposée }\end{array}$ \\
\hline
\end{tabular}


était au collège en classe de quatrième, et à s'opposer à la volonté qu'avaient ses parents, à ce momentlà, de I'y orienter.

Enfin, les aînés jouent parfois un rôle majeur dans I'initiation et l'accompagnement "lectoral » de leurs cadets. C'est le cas dans la famille d'Héléna Chautard (famille biparentale recomposée puis monoparentale, quatre enfants, père jardinier, mère aide-soignante). Benjamine d'une fratrie où les écarts d'âge sont très importants, Héléna a grandi avec deux demi-sœurs et un demi-frère (2). Or, ses deux demi-sœurs ont contribué de façon importante au développement de son intérêt pour la lecture. Toutes les deux aiment, en effet, cette activité, et elles ont incité leur cadette à lire des ouvrages qu'elles avaient appréciés elles-mêmes ( C'est elles qui m'ont poussée, qui m'ont fait lire certains bouquins, qui m'ont fait découvrir des nouveaux livres »). Quand Héléna était enfant, ses demisœurs l'emmenaient à la bibliothèque et lui offraient souvent des livres. Au moment de l'enquête, elles le font d'ailleurs toujours. Cette situation de socialisation " lectorale " par les aînés n'est pas spécifique aux familles nombreuses. Elle se rencontre également souvent dans des familles de taille plus réduite (Court et Henri-Panabière, 2012). Toutefois, la présence de nombreux aînés multiplie les chances de voir l'un d'entre eux jouer ce rôle, notamment parce qu'elle augmente les chances d'avoir un aîné de même sexe que soi. Comme on le verra infra, I'influence que les aînés peuvent exercer sur leurs cadets n'est pas indépendante des caractéristiques de genre des uns et des autres.

Si l'intervention des aînés se limite souvent à un seul domaine (l'encadrement du travail scolaire, l'orientation ou les pratiques « lectorales»), elle se manifeste aussi parfois dans plusieurs domaines simultanément. L'exemple de la famille Bedjaoui (famille biparentale, sept enfants, père ouvrier, mère inactive puis femme de ménage) permet d'illustrer ce cumul. Dans cette famille, où les parents ont suivi des scolarités courtes [M. Bedjaoui a le certificat d'études, sa conjointe a un certificat d'aptitudes professionnelles (CAP)], I'accompagnement scolaire et culturel des enfants repose de manière centrale sur Nora, deuxième enfant de la fratrie et - comme Fatima Kharmaz - aînée des filles. Cette prise en charge par Nora de la scolarité de ses cadets trouve son principe à la fois dans son identité de genre - en tant que fille, elle a été sollicitée précocement par sa mère pour la seconder dans les tâches domestiques et éducatives -, et dans le fait qu'elle a connu un parcours scolaire particulièrement réussi. Alors que son frère aîné a redoublé deux fois, puis a abandonné ses études en première année de CAP, Nora a obtenu de bons résultats tout au long de sa scolarité : elle a sauté la classe de CE1 à l'école primaire, eu les encouragements ou les félicitations chaque année au collège, réussi un baccalauréat littéraire avec mention "bien», et obtenu deux masters (en études théâtrales et en psychologie), là aussi avec la mention «bien ».

D'après son propre récit, Nora est rapidement devenue le "référent-école » de ses petits frères et sœurs. À partir de la fin de sa scolarité primaire, elle les aide dans leurs devoirs, vérifie leurs bulletins et leur donne "des récompenses» lorsqu'ils obtiennent de bons résultats. Au moment où elle s'apprête à quitter le domicile familial, elle anticipe cette séparation en les inscrivant au soutien scolaire assuré par le centre social de leur quartier. Comme Fatima Kharmaz, elle continue à suivre leur scolarité après son départ, en s'informant par téléphone de ce qu'ils font à l'école, et en corrigeant parfois des devoirs envoyés par courriel.

Nora a également joué un rôle décisif dans l'orientation de ses cadets. Selon elle, ses parents ne sont jamais intervenus dans ce domaine, ni pour ellemême ni pour ses frères et sœurs. (" (Ma mère) elle m'a toujours dit: "Tu fais ce que tu veux. L'école, c'est toi qui décides". Si j'avais voulu faire un BEP électricien, elle m'aurait laissé faire un $B E P$ électricien »). Ignorant tout ou presque de la valeur scolaire et sociale des différentes filières, et s'en remettant totalement à l'institution pour ce type de décisions, ils auraient été prêts à accepter l'orientation de leurs enfants dans des filières de relégation, si Nora, mieux informée sur l'organisation du système éducatif, n'était pas intervenue : "Ma sœur Fatiha, raconte-t-elle, ils lui proposaient un BEP sanitaire et social. Alors, à un moment donné, j'ai dit: "Bon, c'est simple, c'est non". Je me suis déplacée au collège, j'ai fait un aller-retour au collège pour avoir un rendez-vous avec le conseiller d'orientation pour lui mettre (les points sur les i) (...) Et pour Halima aussi, tu vois, ils lui ont dit: "La SEGPA (3) c'est super bien". Ma mère, elle y a cru. Là, c'est là où ça a pété, tu vois, j'ai dit : "Arrêtez de déconner, c'est pas possible". (...) I/s ont dit à ma mère : "En sortant de SEGPA, tu peux faire un bac". J'ai une copine qui était au collège avec moi et qui était en SEGPA, elle a passé trois fois son bac. Elle a mon âge, elle a 23 ans, tu vois. Passer trois fois son bac! Elle y arrive pas ». Enfin, Nora a initié et incité ses cadets à la lecture de différentes façons. Quand ils étaient petits, elle leur lisait des contes ou leur racontait des histoires qu'elle inventait elle-même, en s'appliquant à les captiver (" Je leur

(2) Les parents d'Héléna Chautard ont huit enfants, mais Héléna n'a elle-même vécu qu'avec trois germains.

(3) Section d'enseignement général et professionnel adapté. 
racontais des histoires à suite, tu vois, le lendemain : "Voilà ce qu'il se passe". Donc, du coup, ça les tenait »). Plus tard, elle les a régulièrement accompagnés à la bibliothèque municipale et les a inscrits aux activités organisées par les bibliothécaires. Elle leur a également offert des livres "à tous leurs anniversaires".

\section{Les interventions des aînés : une ressource au} féminin

Si l'action des aînés prend des formes différentes en fonction des caractéristiques socioculturelles des familles, elle varie également en fonction du sexe de ces aînés. Qu'ils relaient des parents devenus moins disponibles, ou qu'ils se fassent les interprètes $d^{\prime}$ une culture scolaire avec laquelle les parents ne sont pas familiers, les aînés qui contribuent de façon importante au suivi scolaire et/ou à l'accompagnement « lectoral » de leurs cadets sont en effet avant tout des filles.

Cette prédominance des filles aînées dans l'intervention auprès des cadets est particulièrement nette dans le cas de la lecture. Dans les familles où les aînés ont lu des histoires à leurs cadets, cette pratique est presque toujours le fait des filles (les deux seules fratries où cette pratique est le fait de fils aînés sont composées exclusivement de garçons). Ce rôle des filles aînées en matière de lecture n'est pas étonnant au regard des enquêtes sociologiques sur la lecture. Toutes montrent, en effet, que le goût pour cette activité est plus fréquent chez les filles et chez les femmes (Octobre, 2005 ; Donnat, 2009). Dans la population enquêtée, les filles aînées ont plus souvent que les garçons aînés un goût affirmé pour cette pratique, ce qui leur permet d'envisager la lecture aux cadets comme une activité plaisante. À l'inverse, la plupart des garçons aînés rencontrés au cours de l'enquête n'aiment pas lire. Par exemple, dans la famille Pauvert (famille biparentale, quatre enfants, père chef d'exploitation agricole, mère formatrice), Alexandre (qui est I'aîné de la fratrie) n'a « jamais aimé lire» ("Je lisais (les livres) qu'il fallait lire à l'école puis ça me suffisait. »). Lorsque la benjamine de la fratrie était petite, c'est donc Isabelle (l'aînée des filles) et non Alexandre (I'aîné des garçons) qui lui a lu des histoires, prenant le relais de leur mère qui avait partiellement abandonné cette pratique et, $d^{\prime}$ après $M^{\text {me }}$ Pauvert, elle consacrait beaucoup de temps à cette activité, signe qu'elle la vivait non comme une contrainte, mais comme un plaisir.

Au-delà de ce goût sexuellement différencié pour la lecture, c'est aussi l'assignation des filles à la prise en charge des soins aux jeunes enfants qui explique leur rôle dans l'accompagnement "lectoral » de leurs cadets. Ainsi, dans la famille Bernier (famille biparentale, sept enfants, père directeur d'entreprise, mère kinésithérapeute), Bastien (I'aîné des garçons) aime beaucoup lire, tout comme Marion (I'aînée des filles). Pourtant, Bastien n'a jamais fait la lecture à ses cadets, contrairement à Marion. Cette situation peut s'expliquer par une différence d'implication de ces deux aînés concernant l'aide au travail domestique et aux soins des plus petits. En effet, si Bastien a secondé sa mère dans ces tâches pendant quelques années, il ne l'a plus fait dès l'instant où Marion est devenue assez grande pour endosser ce rôle.

Dans la plupart des familles où la fratrie est mixte, les aînées filles apparaissent également comme plus investies que les aînés garçons dans le suivi scolaire de leurs cadets. Ce sont elles, plus souvent que les garçons, qui surveillent les devoirs de leurs cadets et/ou les aident dans leur travail scolaire, leur parlent de leurs résultats scolaires, les félicitent ou les blâment pour leurs notes. Si certains aînés garçons assurent eux aussi ce suivi du travail et des résultats scolaires de leurs cadets, ils semblent intervenir de manière plus ponctuelle, par exemple en se rendant aux réunions avec les enseignants de leurs germains (ce qu'a fait Mounir, le fils aîné de la famille Kharmaz), en leur donnant des conseils quand ils éprouvent des difficultés (on I'a vu dans le cas de la famille Dallier), ou encore quand ils doivent faire leurs choix d'orientation (on l'a vu dans le cas de la famille Bertou).

Cet investissement plus continu des filles peut s'expliquer à nouveau par leur assignation à la prise en charge des soins aux jeunes enfants. Dans la famille Dumont (famille biparentale, sept enfants, père moniteur d'auto-école, mère sans emploi), I'aînée des filles, Marie-Aline, s'est ainsi beaucoup investie dans le suivi du travail et des résultats scolaires de ses cadets lorsqu'elle était adolescente, et elle continue à le faire au moment de l'enquête quand elle revient au domicile familial pour le week-end ou les vacances : "Ma petite sœur, elle a 9 ans, donc oui, je l'aide à faire ses devoirs le soir quand elle rentre de l'école. Là oui, pour les plus petits j'ai des discussions. Ils me montrent leurs notes et après, voir ce qui a été, ce qui a pas été... ». De manière générale, Marie-Aline s'est beaucoup occupée de ses cadets (elle allait les chercher à l'école, leur faisait faire leurs devoirs, leur faisait prendre leur douche, etc.). Alors que ses parents I'ont sollicitée de manière précoce pour accomplir ces tâches ("Ils me l'ont demandé à partir de mes 7 ans à peu près. Ils me demandaient: "Va faire faire les devoirs à ta sœur" »), ils n'ont pas adressé de demande équivalente à leurs deux fils aînés, pourtant plus âgés qu'elle.

Les différences entre filles et garçons dans le suivi des devoirs des cadets peuvent également s'expliquer par le fait que les aînés garçons mettent en général en œuvre un rapport plus distant aux exigences 
scolaires que les aînées filles [ce qui va dans le même sens que des constats plus généraux sur les différences sexuées entre élèves (Terrail, 1992)]. Peu manifestent la bonne volonté scolaire décrite supra dans le cas de Fatima Kharmaz. Même quand ils sont en situation de réussite scolaire, ces garçons se caractérisent souvent par un rapport instrumental au savoir (et parfois à l'institution scolaire) et par une conformation a minima aux exigences scolaires. François Pradier (famille biparentale, six enfants, père artisan, mère formatrice en éducation civique et religieuse), par exemple, n'a jamais pris plaisir à l'apprentissage des savoirs scolaires, perçus comme " théoriques », " généraux » et « inutiles », et jusqu'à son entrée en BTS, il a toujours fourni le minimum de travail nécessaire au passage dans la classe supérieure (" Jusqu'en terminale, j'aimais pas [l'école], je préférais travailler, je voyais pas l'utilité à long terme »). Cette distance aux savoirs et aux contraintes scolaires se repère également chez de nombreux autres aînés garçons de la population enquêtée, dont Alexandre Pauvert, déjà évoqué, ainsi que Frédéric, I'aîné des garçons dans la famille Wemel.

\section{Les effets bénéfiques de l'intervention des aînés}

\section{Les bénéfices scolaires pour les cadets et leurs limites}

C'est lorsque les résultats scolaires des cadets concernés par l'intervention d'un aîné diffèrent sensiblement de ceux des autres enfants de la fratrie que l'on peut le plus facilement mettre en évidence les effets de cette intervention sur leur scolarité. Dans la famille Kharmaz, les effets positifs de l'intervention éducative de Fatima sont ainsi frappants, puisque les deux derniers enfants de la fratrie (Leïla et Yacine) connaissent de meilleures scolarités que leurs trois aînés. Ces aînés ont, en effet, tous suivi des formations en section professionnelle ou technologique; deux ont redoublé pendant leur scolarité primaire ou secondaire, et aucun n'a fait d'études supérieures. À l'inverse, Yacine (le benjamin) n'a jamais connu de redoublement ; il est « un bon élève jusqu’à présent » et sa sœur Fatima pense qu'il entrera en seconde générale " vu qu'il a les capacités ». Leïla, quant à elle, a toujours été en situation de réussite scolaire. À l'école élémentaire, elle était parmi les premiers de sa classe (elle a failli sauter la dernière année de maternelle car elle savait déjà lire) ; au collège, elle a eu les félicitations chaque année, avant de recevoir une "bourse au mérite " en fin de troisième pour entrer au lycée; elle a ensuite obtenu un baccalauréat économique et social avec mention « assez bien »; enfin, au moment de l'enquête, elle est inscrite à l'université en première année de psychologie, a eu 12,78 de moyenne aux partiels du premier semestre, et envisage de continuer ses études jusqu'au master.
De la même façon, Héléna Chautard (qui est la benjamine et a bénéficié des incitations de ses demisœurs aînées en matière de lecture) a beaucoup mieux réussi scolairement que ses demi-frères et sœurs aînés. De l'école primaire au lycée, elle a toujours eu d'excellents résultats. Elle a notamment sauté deux classes à l'école primaire (le cours préparatoire et le $\mathrm{CM} 1$ ), reçu régulièrement des félicitations, et obtenu le baccalauréat $S$ avec la mention "très bien ». Au moment de l'enquête, elle est inscrite en deuxième année de licence de biologie. Les parcours scolaires de son demi-frère et de ses deux demi-sœurs sont nettement plus moyens puisque tous les trois ont obtenu un baccalauréat technologique ou professionnel et aucun n'a fait d'études supérieures.

L'intervention des aînés n'a, cependant, une influence positive sur la scolarité des cadets que sous certaines conditions. Le poids des identifications de genre constitue une première limite aux effets de ces interventions. Dans le domaine culturel, la différenciation sexuée des pratiques et des goûts des enfants, très sensible en matière de lecture (Octobre, 2005), est en partie liée au fait que ces goûts et ces pratiques se transmettent de manière privilégiée entre un parent et un enfant de même sexe (Henri-Panabière, 2010). Ces transmissions différenciées selon le genre s'observent aussi au sein d'une même fratrie. Ainsi, parmi les benjamins de la famille Kharmaz, c'est Leïla - la seule autre fille de la fratrie - qui a le plus bénéficié de l'intervention de sa sœur aînée. Entre Fatima et Leïla - qui ont partagé la même chambre de la naissance de Leïla au mariage de Fatima - se sont en effet établies des affinités de genre qui permettent d'expliquer, d'une part, l'investissement particulier de Fatima envers sa petite sœur (c'est Fatima qui a convaincu sa mère $d^{\prime}$ avoir un quatrième enfant car elle voulait absolument une petite sœur, et quand celle-ci est née, elle s'est d'emblée beaucoup occupée d'elle) et, d'autre part, la réceptivité particulière de Leïla. Celle-ci a ainsi été plus nettement sensible que son frère Yacine aux incitations "lectorales » de Fatima. Elle appréciait plus que lui qu'on lui lise des histoires et elle a développé un goût plus prononcé pour la lecture.

Les écarts d'âge au sein de la fratrie peuvent également constituer un frein à l'influence des aînés sur les cadets. Lorsque ces écarts sont importants, la durée de cohabitation entre les premiers et les seconds est réduite, les aînés quittant le domicile familial quand leurs benjamins sont encore très jeunes. Le cas de la famille Mejri (famille biparentale recomposée, six enfants, père ouvrier invalide, mère inactive puis employée) permet de l'illustrer. Dans cette fratrie de deux filles et quatre garçons, la benjamine, Nadia, est née six ans après son frère le plus jeune et dix-sept ans après son frère le plus âgé. 
Elle a bénéficié d'un accompagnement scolaire important de la part de ses aînés. À l'instar de ce que I'on observait dans les familles de Nora et de Fatima, c'est avant tout sa sœur aînée, Sarah, qui a pris en charge cet accompagnement. Lorsque Nadia était en primaire, Sarah (qui a onze ans de plus qu'elle) encadrait de près son travail scolaire. Elle surveillait ses devoirs, la "forçait » à faire son cahier de vacances et l'aidait en cas de difficulté. Cet accompagnement a diminué, cependant, dès la fin de l'école primaire. Lorsque Nadia entre en CE2, sa sœur aînée quitte, en effet, le domicile familial pour aller à l'université. Même si elle a continué à suivre le travail de sa cadette à distance (elle revenait régulièrement le week-end et aidait alors Nadia dans ses devoirs), ce suivi s'est fait progressivement plus rare. Après le départ de Sarah, Nadia a cohabité avec trois de ses frères, qui ont pris partiellement le relais de leur sœur aînée (4). Ils aidaient eux aussi leur cadette dans ses devoirs ("Je pouvais compter sur tout le monde», dit Nadia). Ils s'intéressaient également à ses résultats, et la taquinaient à ce propos, créant chez elle une certaine émulation : "Ils trouvaient quand même tous à me dire: "Ah ben moi, j'avais mieux, ah ben moi ci...". Ça c'est normal, moi je fais pareil aujourd'hui avec mes neveux, pour les motiver". Toutefois, ces frères quittent à leur tour le domicile familial quand Nadia a entre 14 ans et 16 ans, de telle sorte que, quand elle entre au lycée, elle vit quasiment comme une fille unique. Or, à cette époque, Nadia commence à éprouver des difficultés scolaires: alors que ses résultats étaient bons jusque-là, elle redouble sa seconde, est orientée en filière technologique contre sa volonté, puis obtient le baccalauréat au rattrapage. L'éloignement de ses aînés a pu contribuer à cet infléchissement de sa scolarité.

\section{Des effets " en retour » : reconnaissance familiale des aînés et répercussions sur les pratiques parentales.}

L'intervention des aînés n'a pas seulement des effets - sous conditions - sur ceux vers lesquels elle se tourne. Elle produit aussi des effets sur les aînés eux-mêmes, et parfois également sur leurs parents. L'investissement des aînés dans l'accompagnement scolaire et « lectoral » de leurs cadets peut favoriser de plusieurs manières leurs propres résultats et parcours scolaires. Le rôle d'un aîné en tant que « référent scolaire » et son statut $d^{\prime}$ « enfant lettré » peuvent tout d'abord être valorisés par l'entourage familial et constituer une "fonction familiale insigne » (Lahire, 1995). L'histoire familiale et les places qu'y prennent les enfants participent à la construction du sens de l'expérience scolaire et des apprentissages (Rochex, 1995). Ce phénomène de valorisation familiale des compétences scolaires des enfants est particulièrement visible dans les familles Bedjaoui et Kharmaz, où la réussite scolaire et la connaissance du système scolaire des aînées sont valorisées, de même que leurs responsabilités familiales en matière de suivi des devoirs et d'orientation des cadets. On peut supposer que la réussite scolaire de ces filles aînées (de Fatima et surtout de Nora) est en partie due au sentiment d'importance que leur confère cette reconnaissance.

Si la reconnaissance symbolique au sein de la famille peut renforcer les dispositions scolaires des aînés, la nature même de leurs pratiques d'accompagnement des cadets et leur mise en œuvre répétée et quotidienne sont également propices à l'acquisition et au renforcement de dispositions scolairement rentables. La pratique régulière et durable de la lecture à voix haute, dès l'école primaire, la fréquentation des bibliothèques, le suivi des devoirs et la vérification du respect des consignes scolaires, la lecture et la correction des écrits et exercices des cadets, constituent autant de manières de se familiariser avec la culture écrite et de renforcer les compétences scolairement acquises en matière de lecture. De plus, le fait de grandir dans une fratrie nombreuse multiplie et diversifie les contextes et les partenaires de sociabilité " lectorale », de même que les niveaux scolaires des cadets suivis, ce qui constitue autant d'occasions d'exercer des savoir-faire «lectoraux» et des connaissances disciplinaires variés. Enfin, I'accompagnement des cadets peut favoriser plus généralement la capacité d'autocontrainte et " l'autonomie » des aînés.

Ces effets sont, là aussi, particulièrement visibles dans le cas de Nora Bedjaoui. Sa fréquentation régulière de la bibliothèque avec ses frères et sœurs a ainsi pu renforcer sa familiarité avec l'univers du livre et ses classements. De même, le fait de prendre en charge la scolarité de ses cadets l'a contrainte à devenir très tôt efficace et autonome dans son propre travail, compétence qu'elle a pu exploiter dans le contexte scolaire, en particulier à l'université ( Moi la fac, j'étais comme un poisson dans l'eau (...), t'es autonome, t'es ton propre chef»), mais aussi au lycée: "Je gérais assez la situation toute seule pour que ma mère ait pas besoin de s'y mêler, elle avait d'autres trucs à faire ».

Enfin, certaines modalités de I'encadrement « lectoral » des cadets peuvent renforcer le goût des aînés pour la lecture en l'associant, dans le contexte affectif familial, à la notion de "plaisir ». Émilie Fernel explique ainsi qu'après avoir profité des histoires lues par sa mère, le fait d'en lire à son tour à ses cadets a constitué « un autre plaisir ».

(4) Le quatrième frère de Nadia est parti de chez leurs parents quand elle était âgée de 3 ans. 
Un dernier effet positif de l'accompagnement scolaire par les aînés réside dans les répercussions qu'il peut avoir sur les manières de faire des parents. Dans certaines familles, tout se passe comme si, en raison de l'intervention des aînées, les parents - et en particulier les mères - avaient appris - ou s'étaient autorisés - à intervenir dans la scolarité de leurs enfants. Ainsi, après son départ du domicile familial, Nora Bedjaoui a insisté auprès de sa mère pour que ses frères et sœurs cadets soient inscrits en soutien scolaire et, de fait, cette dernière s'assure désormais que les collégiens y vont deux fois par semaine. Nora a aussi incité sa mère à récompenser plus souvent les plus jeunes de la fratrie. Nora a ainsi initié les encouragements et les récompenses à ses cadets en cas de bons résultats scolaires (elle a " mis en place un petit peu le truc »: " Je leur payais un paquet de bonbons, ou une connerie, enfin, n'importe quoi mais pour dire: "Bravo, t'as bien fait, c'est cool" »). Nora et sa mère assurant ensemble l'essentiel des tâches domestiques et des soins quotidiens aux enfants, $M^{\text {me }}$ Bedjaoui a régulièrement vu sa fille encadrer ses cadets dans le domaine scolaire et les récompenser, et elle a également subi ses reproches: "Les récompenses, c'est peutêtre venu avec les petits, ma mère elle a appris au fur et à mesure, je lui ai reproché de ne pas m'avoir dynamisée ». On peut aussi supposer que, si Mme Bedjaoui se rend, aujourd'hui, au collège pour rencontrer les professeurs des cadets alors qu'elle ne l'a fait qu'une fois pour Nora, c'est suite aux incitations de celle-ci ("Moi, l'école, elle s'en est jamais vraiment occupée, alors que pour les petits, elle vient aux réunions; moi, elle est jamais venue »).

Dans la famille Kharmaz, on note également une évolution des parents dans leur manière $d^{\prime}$ envisager l'école. Il semble que l'importance qu'ils accordent aux études soit plus forte pour les cadets que pour les aînés, et que l'intervention de ces derniers ait joué un rôle dans cette évolution. Par exemple, M. et $M^{m e}$ Kharmaz ne se sont jamais rendus aux réunions avec les professeurs de leurs aînés mais, lorsque ces derniers ont été assez grands pour les y accompagner, ils ont rencontré les enseignants de leurs cadets et ont ainsi pu se familiariser davantage à l'environnement scolaire de leurs enfants. La présence d'aînés familiers de la culture scolaire a également permis, dans cette famille, de renforcer la surveillance des devoirs des enfants mais aussi l'exercice de l'autorité parentale dans ce domaine. Alors que les parents n'avaient pas les compétences leur permettant de vérifier que les plus grands avaient bien fait leurs devoirs, les aînés ont pu s'acquitter de cette tâche et transmettre cette information à leurs parents, qui peuvent à présent réagir aux devoirs non faits : "Ma mère, raconte Fatima, elle nous grondait, quand on ne faisait pas ses devoirs, mais les petits avantages, c'est que nous (les aînés), même si on faisait pas nos devoirs (...), il suffisait juste de dire :
"Oui, oui, j'ai fait mes devoirs" ; ma mère, elle pouvait pas voir... Alors que, on prend l'exemple de Yacine qui lui fait pas ses devoirs... Même Leïla, vu qu'elle est plus grande que Yacine, elle va venir, et puis elle va le voir et le dire à ma mère. Donc ça fait que, là, automatiquement, il sera puni ». De fait, $M^{\text {me }}$ Kharmaz est plus stricte sur le plan scolaire avec ses cadets qu'avec ses aînés. Elle a instauré pour Yacine (le benjamin) la privation d'ordinateur ou de télévision en cas de devoirs non faits, ce qui n'existait pas pour les aînés.

\section{$\vee \vee \vee$}

\section{Conclusion}

Ainsi, I'intervention des aînés dans l'accompagnement scolaire et « lectoral » de leurs frères et sœurs peut contribuer à forger et à renforcer des dispositions scolairement rentables, et produire des effets positifs sur les parcours scolaires de ces enfants de famille nombreuse. Les effets de cette socialisation fraternelle sont particulièrement sensibles dans les familles de milieux populaires où les parents sont dépourvus de capitaux culturels. Ces aînés, le plus souvent des filles en réussite scolaire, au moins relative, sont, au sein de leur famille, en position d'interprètes de la culture et de l'institution scolaire. Cependant, leur influence ne s'exerce que sous certaines conditions : elle est facilitée par les affinités de genre et elle peut être interrompue en raison d'écarts d'âge importants mettant fin à la cohabitation familiale. Dans les familles scolairement plus dotées, I'action des aînés est moins déterminante, mais elle peut renforcer un mode de socialisation scolairement rentable mis en œuvre initialement par une mère diplômée : les aînées peuvent aller jusqu'à prendre le relais de leur mère dans l'accomplissement de certaines tâches éducatives ou culturelles concernant les cadets. Les parents les plus scolairement et économiquement dotés, dans les classes moyennes et supérieures, comptent, quant à eux, principalement sur d'autres modes d'encadrement (I'autocontrainte précocement acquise par les enfants ou les formes d'encadrement payantes).

Si la recherche montre que l'augmentation du nombre d'enfants pèse sur les conditions de socialisation, en conduisant dans certains cas à un affaiblissement de la disponibilité parentale pour l'accompagnement scolaire et culturel des enfants, et si elle éclaire ainsi les corrélations négatives observées au niveau statistique entre taille de la fratrie et réussite scolaire, son intérêt est aussi de mettre en évidence les ressources et les types d'actions socialisatrices mis en œuvre par les familles nombreuses de différents milieux sociaux pour assurer l'encadrement des enfants, en particulier des derniers-nés. Les interactions entre aînés scolairement dotés et cadets au 
sein de fratries de milieux populaires constituent une ressource spécifique dans le domaine de la familiarisation à la lecture et à la culture scolaire. Cette intervention des aînés a parfois un effet en retour sur leur propre scolarité et constitue donc un facteur de différenciation des parcours scolaires au sein d'une même fratrie. La recherche souligne ainsi la nécessité de prendre en compte les relations entre frères et sœurs dans l'analyse des configurations familiales et de leurs effets.

\section{\begin{tabular}{|l|l}
\hline & \\
\hline Références & bibliographiques
\end{tabular}}

- Barnet-Verzat C. et Wolff F.-C., 2003, Choix d'éducation et composition par sexe de la fratrie, Économie et Prévision, $\mathrm{n}^{\circ}$ 157:97-118.

- Bertrand J., Bois G., Court M., Henri-Panabière G. et Vanhée O., 2012 a, Scolarité dans les familles nombreuses populaires et conditions matérielles d'existence, Informations sociales, n 173:74-82.

- Bertrand J., Bois G., Court M., Henri-Panabière G. et Vanhée O., 2012 b, « Les parcours scolaires d'enfants de familles nombreuses: I'analyse des processus de socialisation », rapport de recherche pour la Caisse nationale des Allocations familiales, Centre Max Weber, université Lyon 2.

- Blanpain N., 2007, "Les conditions de vie des familles nombreuses », Études et Résultats, $n^{\circ} 155$.

- Caillé J.-P. et O'Prey S., 2002, Les familles immigrées et l'école française : un rapport singulier qui persiste même après un long séjour en France, INSEE, Données sociales:149-159.

- Caillé J.-P. et Rosenwald F., 2006, "Les inégalités de réussite à l'école élémentaire : construction et évolution », INSEE, France, portrait social:115-137.

- Chauvel L., 2001, Le retour des classes sociales, Revue de l'OFCE, nº 79:315-359.

- Coulangeon P., 2007, Lecture et télévision. Les transformations du rôle culturel de l'école à l'épreuve de la massification scolaire, Revue française de sociologie, vol. 48, n 4:657-691.

- Court M. et Henri-Panabière G., 2012, La socialisation culturelle au sein de la famille: le rôle des frères et sœurs, Revue française de pédagogie, $\mathrm{n}^{\circ}$ 179:5-13.

- Donnat O., 2009, Les Pratiques culturelles des Français à l'ère numérique - Enquête 2008, Paris, La Découverte/Ministère de la Culture et de la Communication.

- Henri-Panabière G., 2010, Des « héritiers » en échec scolaire, Paris, La Dispute.

- Héran F., 1994, "L'aide au travail scolaire : les mères persévèrent ", Insee Première, n 350.

- Lahire B., 1995, Tableaux de familles. Heurs et malheurs scolaires en milieux populaires, Paris, Gallimard/Le Seuil (réédition en poche, Points, 2012).

- Lahire B., 1992, L'inégalité devant la culture écrite scolaire : le cas de l'« expression écrite » à l'école primaire, Sociétés contemporaines, $\mathrm{n}^{\circ}$ 11:171-191.

- Laurens J.-P., 1992, 1 sur 500. La réussite scolaire en milieu populaire, Toulouse, Presses universitaires du Mirail.

- Michaudon H., 2001, "La lecture, une affaire de famille », INSEE Première, $n^{\circ} 777$.

- Octobre S., 2005, "La fabrique sexuée des goûts culturels. Construire son identité de fille ou de garçon à travers les activités culturelles", Développement culturel, $\mathrm{n}^{\circ} 150$.

- Poullaouec T., 2010, Le diplôme, l'arme des faibles, Paris, La Dispute, collection L'enjeu scolaire.

- Renard F., 2008, Une approche sociologique des habitudes de lecture. Pour une étude conjointe des contextes scolaire et extrascolaire, Éducation \& Didactique, vol. 2, $\mathrm{n}^{\circ}$ 1:41-68.

- Rochex J.-Y., 1995, Le sens de l'expérience scolaire, Paris, Presses universitaires de France.

- Terrail J.- P., 1992, Parents, filles et garçons face à l'enjeu scolaire, Éducation et Formations, n 30:3-11. 
